\title{
Deep sequencing reveals clonal evolution patterns and mutation events associated with relapse in B-cell lymphomas
}

\author{
Yanwen Jiang ${ }^{1,2}$, David Redmond ${ }^{1}$, Kui Nie ${ }^{3}$, Ken W Eng ${ }^{1}$, Thomas Clozel ${ }^{4}$, Peter Martin², Leonard HC Tan ${ }^{5}$,
} Ari M Melnick', Wayne Tam ${ }^{3^{*}}$ and Olivier Elemento ${ }^{1^{*}}$

\begin{abstract}
Background: Molecular mechanisms associated with frequent relapse of diffuse large B-cell lymphoma (DLBCL) are poorly defined. It is especially unclear how primary tumor clonal heterogeneity contributes to relapse. Here, we explore unique features of B-cell lymphomas - VDJ recombination and somatic hypermutation - to address this question.

Results: We performed high-throughput sequencing of rearranged VDJ junctions in 14 pairs of matched diagnosis-relapse tumors, among which 7 pairs were further characterized by exome sequencing. We identify two distinctive modes of clonal evolution of DLBCL relapse: an early-divergent mode in which clonally related diagnosis and relapse tumors diverged early and developed in parallel; and a late-divergent mode in which relapse tumors developed directly from diagnosis tumors with minor divergence. By examining mutation patterns in the context of phylogenetic information provided by VDJ junctions, we identified mutations in epigenetic modifiers such as KMT2D as potential early driving events in lymphomagenesis and immune escape alterations as relapse-associated events.

Conclusions: Altogether, our study for the first time provides important evidence that DLBCL relapse may result from multiple, distinct tumor evolutionary mechanisms, providing rationale for therapies for each mechanism. Moreover, this study highlights the urgent need to understand the driving roles of epigenetic modifier mutations in lymphomagenesis, and immune surveillance factor genetic lesions in relapse.
\end{abstract}

\section{Background}

Diffuse large B-cell lymphoma (DLBCL) is an aggressive form of non-Hodgkin's lymphoma in which one-third of patients either do not respond to initial therapy or relapse after standard therapy, such as dose-dense or standard immunochemotherapy with rituximab and cyclophosphamide, doxorubicin, vincristine, and prednisone (R-CHOP) [1]. Although relapses normally occur early (first 2 to 3 years), some do occur after 5 years [2]. Treatment options for relapse and refractory DLBCLs are limited and only $10 \%$ of the relapsed patients achieve 3-year progression-free survival following these treatments,

\footnotetext{
* Correspondence: wtam@med.cornell.edu; ole2001@med.cornell.edu ${ }^{3}$ Department of Pathology and Laboratory Medicine, Weill Cornell Medical College, New York, NY 10021, USA

'Institute for Computational Biomedicine, Weill Cornell Medical College, New York, NY 10021, USA

Full list of author information is available at the end of the article
}

underlying the urgent need for novel approaches to treat DLBCL relapse $[3,4]$. Unfortunately, our current understanding of the molecular mechanisms associated with DLBCL relapse is limited. It is, for example, unclear whether genetic mutations present in the tumor at diagnosis or acquired after treatment help certain DLBCL cell populations to acquire resistance to treatment. More generally, it is presently unclear whether clonal heterogeneity in primary tumors plays a role in DLBCL relapse.

Previously, Ding et al. [5] examined genetic changes associated with acute myeloid leukemia relapse using whole-genome sequencing and used patterns of mutational abundances to infer clonal evolution patterns. However, due to the lack of tractable markers on myeloid cells, it is difficult to precisely and definitely deduce clonal evolution patterns between diagnosis and relapse acute myeloid leukemias. The situation is potentially different in lymphoid malignancies originating from mature $\mathrm{B}$ cells, since the 
latter harbor a natural clonality marker in the form of VDJ (or VJ) junctions. Indeed, in order to generate a diversified repertoire of antibodies, each $B$ cell undergoes somatic immunoglobulin heavy chain (IGH) VDJ recombination at the pro B-cell stage to create a single productive VDJ junction from a large pool of $V_{H}$ (variable), $D$ (diversity), and $\mathrm{J}_{\mathrm{H}}$ (joining) segments. In addition, during this process, non-templated nucleotides (N-bases) may be added at the junctions, and a small part of the $\mathrm{V}_{\mathrm{H}}, \mathrm{D}, \mathrm{J}_{\mathrm{H}}$ germline sequences may be deleted, resulting in a unique VDJ rearrangement, which effectively tags each $B$ cell and its progeny [6]. In the subsequent germinal center (GC) reaction, $\mathrm{B}$ cells further introduce point mutations into the recombined VDJ sequences, a process known as somatic hypermutation (SHM), to enhance antibody affinity $[7,8]$. Because DLBCLs arise from GC or post-GC B cells, we hypothesized that their clonal populations can be tracked by their VDJ and SHM patterns to reveal valuable information regarding intra-tumor heterogeneity and clonal evolution of the disease. To test this hypothesis, we performed high-throughput sequencing of rearranged VDJ junctions in 14 pairs of matched primary diagnosisrelapse DLBCLs and discovered two distinct clonal evolutionary scenarios of DLBCL relapse. Furthermore, in conjunction with exome sequencing on several diagnosis-relapse pairs, we identified mutations within histone-modifying enzymes as candidate early drivers in DLBCL lymphomagenesis, and genetic lesions in immune surveillance genes as potential facilitators in DLBCL relapse.

\section{Results}

\section{Deep VDJ sequencing revealing clonal heterogeneity of DLBCL}

To trace the clonal identities of the diagnosis-relapse DLBCL pairs $(\mathrm{N}=14$, patient information summarized in Additional file 1), we performed Illumina MiSeq PE $2 \times 150$ bp sequencing on the 300 to $350 \mathrm{bp} \mathrm{PCR}$ products targeting the genomic IGH VDJ sequences (see Materials and methods). We reasoned that long reads generated using this approach should enable phylogenetic analysis based on SHM mutation patterns. We generated 0.38 to 1.42 million paired-end reads/sample (average $0.75 \pm 0.26$ million; Additional file 2). We developed a custom bioinformatics pipeline to align each paired-end read to germline immunoglobulin $\mathrm{V}, \mathrm{D}$, and $\mathrm{J}$ sequences in the IMGT database [9] and annotate the recombined VDJ junctions (see Materials and methods). The average alignment rate was $67.7 \pm 14.1 \%$ (Additional file 2). In total, we identified 0.28 to 0.93 million (average $0.49 \pm 0.15$ million) $\mathrm{V}_{\mathrm{H}} \mathrm{DJ} \mathrm{H}_{\mathrm{H}}$ junctions per sample (Additional file 2), and the numbers of aligned reads were comparable between diagnosis and relapse samples within each pair (Figure S1 in Additional file 3).
We first sought to examine the $\mathrm{V}_{\mathrm{H}} \mathrm{DJ}$ heterogeneity of each individual tumor (diagnosis or relapse) and counted how many times each combination of $\mathrm{V}_{\mathrm{H}}, \mathrm{D}$, and $\mathrm{J}_{\mathrm{H}}$ was found. We found, on average, 7.4 distinct $\mathrm{V}_{\mathrm{H}} \mathrm{DJ} \mathrm{H}_{\mathrm{H}}$ junctions per thousand mapped paired-end reads per sample (Table 1). However, the number of unique rearrangements per sample ranged broadly from 0.5 to 27.1 per thousand mapped paired-end reads, indicating high VDJ heterogeneity within DLBCL samples, similar to what has been reported in acute B lymphoblastic leukemia (B-ALL) [10]. There was no significant difference in number of unique rearrangements per thousand mapped paired-end reads between the paired diagnosis and relapse samples (6.6 \pm 7.5 versus $6.2 \pm 6.9, P=0.85$, paired $t$-test; or $P=0.80$, Wilcoxon matched pairs test; Figure S2 in Additional file 3). For each sample, there was either one or two dominant $\mathrm{V}_{\mathrm{H}} \mathrm{DJ}_{\mathrm{H}}$ rearrangements that together account for $65 \%$, on average, of all the identified $\mathrm{V}_{\mathrm{H}} \mathrm{DJ} \mathrm{H}_{\mathrm{H}}$ sequences, likely representing the productive and nonproductive alleles of the major tumor subclone [11] (Table 1). The other less abundant $\mathrm{V}_{\mathrm{H}} \mathrm{DJ}_{\mathrm{H}}$ rearrangements were likely representing either $\mathrm{V}_{\mathrm{H}} \mathrm{DJ} \mathrm{J}_{\mathrm{H}}$ sequences with high SHM rates that could not be readily mapped to major tumor $\mathrm{V}_{\mathrm{H}} \mathrm{DJ}_{\mathrm{H}}$ sequences, or benign B-cell infiltration within the DLBCL tumors. To further address this question, we decided to compare the clonal distribution frequency between the tumor and normal B-cell populations by performing VDJ sequencing on a bone marrow (BM) B-cell sample and tumor sample from one DLBCL patient. Although tumor cell infiltration was observed in the BM sample (approximately $1.1 \%$ of the total $\mathrm{V}_{\mathrm{H}} \mathrm{DJ} \mathrm{J}_{\mathrm{H}}$ sequences), most $\mathrm{V}_{\mathrm{H}} \mathrm{DJ} \mathrm{H}_{\mathrm{H}}$ sequences did not match the tumor $\mathrm{V}_{\mathrm{H}} \mathrm{DI}$ H and therefore correspond to normal non-malignant $\mathrm{B}$ cells. We observed that the major dominant $\mathrm{V}_{\mathrm{H}} \mathrm{DJ} \mathrm{J}_{\mathrm{H}}$ rearrangements only accounted for $1.5 \%$ in the BM sample, similar to what has been reported previously for normal B cells [12]. Thus, the dominant $\mathrm{V}_{\mathrm{H}} \mathrm{DJ} \mathrm{H}_{\mathrm{H}}$ rearrangements we observed in tumor samples are not compatible with normal B-cell clonal expansion and indeed represent the malignant B-cell populations in these tumors. Furthermore, when we excluded the dominant $\mathrm{V}_{\mathrm{H}} \mathrm{DJ} \mathrm{J}_{\mathrm{H}}$ rearrangements from the tumor sample (the tumor rearrangement) and then compared the distribution of the remaining $\mathrm{V}_{\mathrm{H}} \mathrm{DJ}_{\mathrm{H}}$ rearrangements of the tumor sample to the BM sample, we observed similar distribution frequencies (Figure S3 in Additional file 3), indicating that these minor $\mathrm{V}_{\mathrm{H}} \mathrm{DJ}$, rearrangement clones were indeed likely representing infiltrating non-malignant normal B-cell populations $\left(\mathrm{V}_{\mathrm{H}} \mathrm{DJ} \mathrm{H}_{\mathrm{H}}\right.$ sequence mapping errors due to high SHM rates cannot be completely excluded). The diagnosis and relapse tumors of all but one pair (pair 6) harbored the same major $\mathrm{V}_{\mathrm{H}} \mathrm{DJ} \mathrm{H}_{\mathrm{H}}$ rearrangement, demonstrating that they were clonally related regardless of the length of time it took the relapse to develop (Table 1). Accordingly, the relapse tumors of late-relapse 
Table 1 Summary of $V_{H} D J_{H}$ sequencing results

\begin{tabular}{|c|c|c|c|c|c|c|c|c|}
\hline Pair number & $\begin{array}{l}\text { Sample } \\
\text { ID }\end{array}$ & Diagnosis/relapse & $\begin{array}{c}\text { Number of unique } \\
V_{H} D J_{H} \text { rearrangements }\end{array}$ & $\begin{array}{c}\text { Number of unique } \\
V_{H} D J_{H} \text { rearrangement } \\
\text { per } 10^{3} \text { mapped reads }\end{array}$ & Dominant $\mathrm{V}_{\mathrm{H}} \mathrm{DJ} \mathrm{H}_{\mathrm{H}}$ rearrangement & $\begin{array}{l}\text { Dominant } \\
\text { rearrangement } \\
\text { percentage }\end{array}$ & $\begin{array}{c}\text { Number of } \\
\text { sub-clones } \\
(>10 X) \text { within } \\
\text { dominant } V_{H} \mathrm{JJ}_{\mathrm{H}}\end{array}$ & $\begin{array}{c}\text { Number of } \\
\text { sub-clones }(>10 \mathrm{X}) \\
\text { per } 10^{3} \text { dominant } \\
\mathrm{V}_{\mathrm{H}} \mathrm{D} \mathrm{J}_{\mathrm{H}} \text { alignments } \\
\end{array}$ \\
\hline \multirow[t]{4}{*}{1} & $1 \mathrm{D}$ & Diagnosis & 311 & 0.69 & IGHV4-34 IGHD3-22 IGHJ5 & $96.9 \%$ & 1,591 & 3.60 \\
\hline & $1 \mathrm{R} 1$ & Relapse & 265 & 0.50 & IGHV4-34 IGHD3-22 IGHJ5 & $94.9 \%$ & 2,109 & 4.07 \\
\hline & $1 R 2$ & Relapse & NA & NA & NA & NA & NA & NA \\
\hline & 1R3 & Relapse & 396 & 0.66 & IGHV4-34 IGHD3-22 IGHJ5 & $94.5 \%$ & 2,487 & 4.26 \\
\hline \multirow[t]{3}{*}{2} & $2 \mathrm{D}$ & Diagnosis & 315 & 0.72 & IGHV4-59 IGHD6-19 IGHJ5 & $82.2 \%$ & 1,963 & 4.53 \\
\hline & $2 \mathrm{R} 1$ & Relapse & 3,131 & 7.27 & IGHV4-59 IGHD6-19 IGHJ5 & $88.9 \%$ & 1,372 & 3.44 \\
\hline & $2 \mathrm{R} 2$ & Relapse & NA & NA & NA & NA & NA & NA \\
\hline \multirow[t]{4}{*}{3} & $3 \mathrm{D} 1$ & Diagnosis & 950 & 1.84 & IGHV3-49 IGHD2-8 IGHJ4 & $28.3 \%$ & 421 & 2.81 \\
\hline & & & & & IGHV3-49 IGHD3-22 IGHJ4 & $27.0 \%$ & 596 & 2.13 \\
\hline & $3 R 2$ & Relapse & 745 & 1.29 & IGHV3-49 IGHD3-22 IGHJ4 & $29.2 \%$ & 608 & 2.41 \\
\hline & & & & & IGHV3-49 IGHD2-8 IGHJ4 & $28.0 \%$ & 781 & 3.17 \\
\hline \multirow[t]{2}{*}{4} & $4 \mathrm{D}$ & Diagnosis & 9,158 & 23.08 & IGHV3-7 IGHD4-17 IGHJ6 & $69.7 \%$ & 1,318 & 4.75 \\
\hline & $4 \mathrm{PR}$ & Progression & 1,785 & 4.34 & IGHV3-7 IGHD4-17 IGHJ6 & $43.0 \%$ & 933 & 5.23 \\
\hline \multirow[t]{4}{*}{5} & $9 D$ & Diagnosis & 2,911 & 4.27 & IGHV4-34 IGHD6-13 IGHJ6 & $47.5 \%$ & 1,381 & 4.16 \\
\hline & & & & & IGHV1-18 IGHD6-13 IGHJ2 & $39.6 \%$ & 1,075 & 3.96 \\
\hline & $9 R$ & Relapse & 18,683 & 25.72 & IGHV1-18 IGHD6-13 IGHJ2 & $9.2 \%$ & 328 & 4.57 \\
\hline & & & & & IGHV4-34 IGHD6-13 IGHJ6 & $3.5 \%$ & 154 & 5.33 \\
\hline \multirow[t]{2}{*}{6} & $12 \mathrm{D}$ & Diagnosis & 7,570 & 22.12 & IGHV3-74 IGHD4-17 IGHJ4 & $25.2 \%$ & 0 & 0 \\
\hline & $12 \mathrm{R}$ & Relapse & 7,299 & 13.78 & IGHV3-23 IGHD6-13 IGHJ4 & $24.1 \%$ & 496 & 3.86 \\
\hline \multirow[t]{3}{*}{7} & 13D1 & Diagnosis & 7,931 & 27.09 & IGHV3-23 IGHD3-9 IGHJ6 & $82.4 \%$ & 870 & 5.14 \\
\hline & $13 \mathrm{D} 2$ & Diagnosis & 2,436 & 6.65 & IGHV3-23 IGHD3-9 IGHJ6 & $89.6 \%$ & 718 & 5.74 \\
\hline & $13 R$ & Relapse & 1,204 & 3.71 & IGHV3-23 IGHD3-9 IGHJ6 & $93.1 \%$ & 858 & 5.27 \\
\hline \multirow[t]{4}{*}{8} & $14 \mathrm{D}$ & Diagnosis & 4,172 & 12.07 & IGHV1-18 IGHD6-13 IGHJ2 & $48.0 \%$ & 819 & 4.89 \\
\hline & & & & & IGHV4-34 IGHD6-13 IGHJ6 & $24.1 \%$ & 327 & 3.76 \\
\hline & $14 \mathrm{R}$ & Relapse & 1,206 & 3.84 & IGHV1-18 IGHD6-13 IGHJ2 & $57.7 \%$ & 883 & 4.81 \\
\hline & & & & & IGHV4-34 IGHD6-13 IGHJ6 & $32.1 \%$ & 421 & 3.96 \\
\hline \multirow[t]{5}{*}{9} & 15D1 & Diagnosis & 8,727 & 15.47 & IGHV1-18 IGHD6-13 IGHJ2 & $23.7 \%$ & 471 & 4.97 \\
\hline & & & & & IGHV3-48 IGHD2-2 IGHJ4 & $26.8 \%$ & 0 & 0 \\
\hline & $15 \mathrm{D} 2$ & Diagnosis & 955 & 2.83 & IGHV1-18 IGHD6-13 IGHJ2 & $37.3 \%$ & 18 & 2.73 \\
\hline & & & & & IGHV4-34 IGHD6-13 IGHJ6 & $35.4 \%$ & 0 & 0 \\
\hline & $15 \mathrm{R}$ & Relapse & 1,296 & 4.62 & IGHV3-48 IGHD2-2 IGHJ4 & $37.1 \%$ & 530 & 4.95 \\
\hline
\end{tabular}


Table 1 Summary of $\mathbf{V}_{\mathbf{H}} \mathbf{D} \mathbf{J}_{\mathbf{H}}$ sequencing results (Continued)

\begin{tabular}{|c|c|c|c|c|c|c|c|c|}
\hline & & & & & IGHV1-18 IGHD6-13 IGHJ2 & $33.5 \%$ & 471 & 5.00 \\
\hline \multirow[t]{2}{*}{10} & $16 \mathrm{D}$ & Diagnosis & 11,303 & 12.10 & IGHV1-18 IGHD6-13 IGHJ2 & $55.2 \%$ & 867 & 1.61 \\
\hline & $16 R$ & Relapse & 1,875 & 2.67 & |GHV1-18 IGHD6-13 IGHJ2 & $44.8 \%$ & 1,079 & 3.42 \\
\hline \multirow[t]{2}{*}{11} & F6D & Diagnosis & 786 & 1.04 & IGHV3-23 IGHD3-22 IGHJ4 & $72.5 \%$ & 991 & 4.22 \\
\hline & F6PR & Progression & 2,135 & 4.03 & IGHV3-23 IGHD3-22 IGHJ4 & $61.0 \%$ & 1,245 & 5.29 \\
\hline \multirow[t]{2}{*}{12} & F7D & Diagnosis & 3,491 & 6.07 & IGHV4-34 IGHD3-22 IGHJ6 & $45.2 \%$ & 231 & 0.61 \\
\hline & F7R & Relapse & 6,115 & 14.58 & IGHV4-34 IGHD3-22 IGHJ6 & $40.0 \%$ & 82 & 0.33 \\
\hline \multirow[t]{3}{*}{13} & SPF6-1 & Diagnosis & 1,160 & 2.41 & IGHV3-7 IGHD3-10 IGHJ4 & $90.0 \%$ & 1,691 & 3.83 \\
\hline & SPF6-2 & Diagnosis & 828 & 1.75 & IGHV3-7 IGHD3-10 IGHJ4 & $93.1 \%$ & 1,854 & 3.93 \\
\hline & SPF6-3 & Relapse & 719 & 1.54 & IGHV3-7 IGHD3-10 IGHJ4 & $95.8 \%$ & 1,754 & 3.85 \\
\hline \multirow[t]{2}{*}{14} & SPF10-1 & Diagnosis & 1,463 & 3.17 & IGHV3-49 IGHD3-10 IGHJ5 & $67.8 \%$ & 1,425 & 3.65 \\
\hline & SPF10-2 & Relapse & 1,731 & 3.73 & IGHV3-49 IGHD3-10 IGHJ5 & $30.2 \%$ & 777 & 4.37 \\
\hline
\end{tabular}

NA, not available. 
patients who developed relapse disease 5 to 10 years after initial diagnosis (pairs 1, 8, 9, 12; Additional file 1) still carried the same $\mathrm{V}_{\mathrm{H}} \mathrm{DJ} \mathrm{J}_{\mathrm{H}}$ rearrangements as their respective diagnosis tumors.

\section{Two scenarios of DLBCL relapse clonal evolution}

We further focused our analysis on the major $\mathrm{V}_{\mathrm{H}} \mathrm{DJ} \mathrm{J}_{\mathrm{H}}$ rearrangement identified in each tumor. In addition to $\mathrm{V}_{\mathrm{H}} \mathrm{DJ}_{\mathrm{H}}$ rearrangement, DLBCL cells have undergone various degrees of SHM within the rearranged $\mathrm{V}_{\mathrm{H}} \mathrm{DJ}_{\mathrm{H}}$ sequences, which can in theory be further utilized to delineate the subclonal population structure of the tumor. Indeed, we identified unique subclones (found at least 10 times) with distinctive SHM patterns within the dominant $\mathrm{V}_{\mathrm{H}} \mathrm{DJ}_{\mathrm{H}}$ sequence of each sample (Table 1). Diagnosis samples had, on average, $3.6 \pm 1.4$ subclones per thousand mapped major $\mathrm{V}_{\mathrm{H}} \mathrm{DJ}_{\mathrm{H}}$ rearrangements. Relapse tumors had $4.1 \pm 1.3$ subclones per thousand mapped major $\mathrm{V}_{\mathrm{H}} \mathrm{DJ}_{\mathrm{H}}$ rearrangements. There was no significant difference in the subclone frequency between the relapse and diagnosis samples ( $P=0.07$, two-tailed paired $t$-test; or $P=0.17$, Wilcoxon matched pairs test; Figure S4 in Additional file 3). We then observed that eight pairs of samples had increased normalized subclone numbers in relapse samples, while five pairs had decreased normalized subclone numbers in relapse samples compared with their respective diagnosis samples (Figure S4 in Additional file 3; we excluded pair 6 in this and subsequent VDJ subclone analyses due to unmatched dominant VDJ between diagnosis and relapse samples), suggesting that the clonal subpopulation change is a dynamic process during DLBCL relapse.

We then sought to use the subclone SHM information to trace the clonal evolution of each sample pair. As described in Materials and methods, we performed phylogenetic analysis of the SHM profiles of the major $\mathrm{V}_{\mathrm{H}} \mathrm{DJ}_{\mathrm{H}}$ rearrangements between each diagnosis and relapse pair using the neighbor joining phylogenetic tree reconstruction approach [13]. This analysis uncovered two distinct scenarios of DLBCL relapse (Figure 1A; Figure S5 in Additional file 3). In one scenario (scenario I, $\mathrm{n}=6$, pairs $1,2,4,5,9,10$ ), the major relapse clones clustered in a separate branch from the diagnosis clones on the phylogenetic tree (Figure 1A; Figure S5A,B,D,E,H,I in Additional file 3). The relapse clones clustered either alone $(n=2$, pairs 4 and 9) or together with a highly divergent minor diagnosis clone ( $\mathrm{n}=4$; Figure 1A; see the small blue clone (third bar above the major relapse clone) clustered together with the red clones within the top branch). This particular pattern indicates that, in this scenario, although the relapse and diagnosis tumors were derived from the same B cell (they share the same $\mathrm{V}_{\mathrm{H}} \mathrm{DJ} \mathrm{J}_{\mathrm{H}}$ rearrangement and several somatic hypermutations), their precursors have diverged, acquired different mutations within $\mathrm{V}_{\mathrm{H}} \mathrm{DJ}_{\mathrm{H}}$ sequences (Figure 1A, right panel), and expanded at different times. Moreover, that the major relapse clones frequently cluster with a minor divergent clone found at diagnosis suggest that preexisting, chemoresistant and divergent diagnosis subclones are capable of eventually regenerating entire relapse tumors.

In the second scenario (scenario II, $\mathrm{n}=7$, pairs $3,7,8$, $11,12,13,14)$, the dominant diagnosis and relapse clones clustered together very closely (Figure 1B, left panel), with similar SHM patterns. The dominant clones in the diagnosis and relapse tumor share the vast majority of the IgVH somatic mutations, with only one or few different mutations in the relapse tumor that could not differentiate them into separate branches (Figure 1B, right panel; Figure S5C,F-G,J-M in Additional file 3). This observation is compatible with a scenario where the relapse tumors arise linearly from the major diagnosis clone or from a highly related subclone that appears to be more abundant in the diagnosis tumor compared with the former scenario.

Due to unequal starting amounts of DNA, our VDJ sequencing libraries produced variable numbers of reads as indicated in the previous section. To make sure that VDJ sequencing depth would not affect our clonal evolution analysis, we sequenced two pairs of samples (one from scenario I (pair 10) and one from scenario II (pair 3)) to a very high depth (approximately two million paired reads per sample) compared with our original analysis, and then systematically subsampled the reads with decreasing rates (1/10th each round) to assess the VDJ recombination composition. We found that although the major VDJ frequency remained the same across all sample rates and for each sample (Figure S6A in Additional file 3), we did observe increased numbers of subclones with increasing sequencing depth (Figure S6B in Additional file 3). When we constructed phylogenetic trees of the subclones discovered at different subsampling levels for these two samples, we found that the relapse clonal evolution patterns did not change with increased number of subclones between the range of 10,000 to 1,000,000 reads (Figure S6C in Additional file 3), within which our original VDJ sequencing depth occurred, suggesting that the slight difference in VDJ sequencing depth across our samples would not undermine the accuracy of our relapse clonal evolution analysis.

We next examined the mutational distance between the major clones within the diagnosis and the relapse pairs. We found that divergent scenario (scenario I) diagnosis-relapse pairs had significantly more SHMs in the relapse samples and the SHMs occurred at different sites compared with diagnosis samples $(P=0.03$, Wilcoxon matched pairs test). On the other hand, sample pairs of the less divergent scenario (scenario II) had less difference in SHM number and location between the relapse and diagnosis when compared with the divergent 


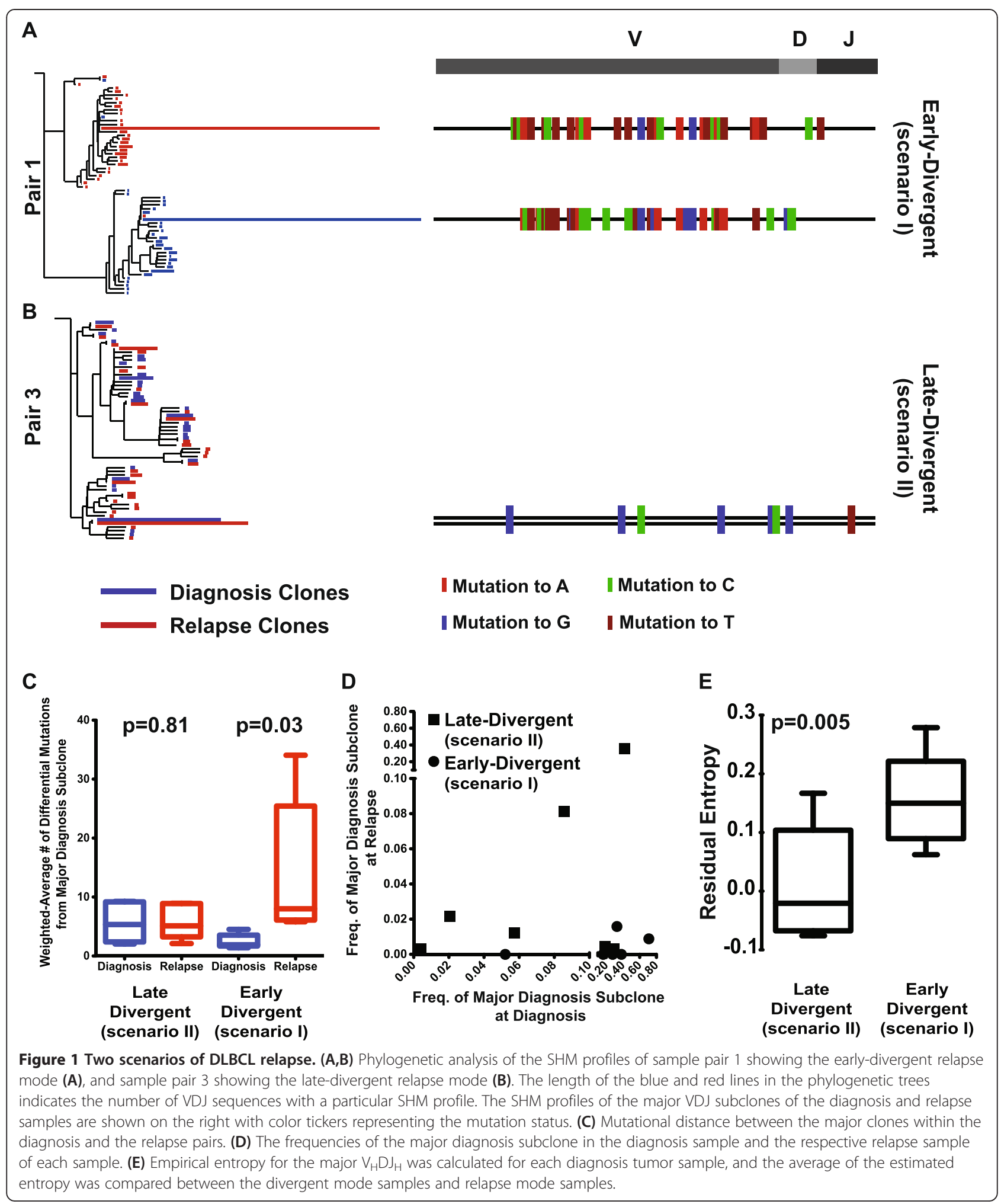

scenario pairs $(P=0.81$, Wilcoxon matched pairs test; Figure $1 C$ ).

Moreover, when we traced the fate of the major diagnosis subclone within the relapse tumor, we found that they almost disappeared in the relapse tumors of the more divergent mode pairs (scenario I; Figure 1D). On the contrary, the major diagnosis subclone maintained the same relative abundance in the relapse tumors of the less divergent cases 
(scenario II; Figure 1D). Surprisingly, we noticed a couple of the less divergent cases (scenario II) behaved similarly to the more divergent cases (scenario I), namely sample pairs 7 and 13 (Figure 1D). We plotted the relapse subclone frequencies according to their distance from the major diagnosis subclones (number of SHMs different from the major diagnosis subclone) and found that, in these two pairs, the entire major relapse subclones differed in only one or two mutations compared with their respective diagnosis subclones (Figure S7 in Additional file 3). Therefore, the abundance of the major diagnosis subclone decreased to almost 0 at relapse in these samples. We performed the same analysis on the more divergent sample pairs (scenario I), and found that the difference in SHM pattern between the major diagnosis and relapse subclones was much greater (pairs 2 and 9, for example; Figure S7 in Additional file 3).

Based on these VDJ SHM analyses, two patterns emerge that differ in the degree of divergence (from the major diagnosis subclones) of the tumor clones from which the relapse tumors were derived. The first mode (scenario I) demonstrates a distinct divergence; the diverged subclone is present as a very minor subclone in the diagnosis tumor in some cases and appears to arise earlier in the diagnosis tumor during tumor evolution. We termed scenario I the early-divergent mode. The second mode (scenario II) does not show a distinct divergence and suggests that the relapse tumor may be derived from a subclone that is more closely related to the major diagnosis subclone (often more abundant) at a relatively late stage during tumor evolution. We therefore named scenario II the late-divergent mode. The presence of two modes of relapse, early-divergent (scenario I) or late-divergent (scenario II), can be further confirmed by whole exome sequencing (see below).

\section{Diagnosis tumors with early-divergent evolution to relapse show increased clonal heterogeneity compared with tumors with late-divergent evolution}

We examined whether the two relapse modes identified in this study were correlated with clinical features of DLBCL. By performing immunohistochemistry on BCL6, CD10, and MUM1 (Hans classification), we were able to determine the subtypes of these samples (GCB-DLBCL versus non-GCB-DLBCL; Additional file 1). We found the time to relapse was significantly higher in GCB-DLBCL compared with non-GCB-DLBCL $(P=0.02$, MannWhitney test). However, when we examined the relationship between disease subtypes and relapse modes, we found that both subtypes were evenly distributed between the two relapse modes (chi-square test $P=0.55$ ), indicating that there is no correlation between DLBCL subtypes and relapse clonal evolution. We also compared the average time to relapse and did not find significant difference between the two modes of relapse $(3.2 \pm 1.5$ years versus $3.0 \pm 1.0$ years, late-divergent versus early-divergent, $P=0.74$, Mann-Whitney test). We then investigated whether clonal heterogeneity at diagnosis could predict which scenario towards relapse (early or late divergent) a patient was most likely to follow. We examined the pattern of subclones with distinctive SHM patterns within the dominant $\mathrm{V}_{\mathrm{H}} \mathrm{DJ} \mathrm{H}_{\mathrm{H}}$ sequence of each sample, and used the frequency with which each subclone appeared to calculate the empirical entropy for the major $\mathrm{V}_{\mathrm{H}} \mathrm{DJ}_{\mathrm{H}}$ of each diagnosis tumor sample. Empirical entropy measures the diversity of SHM patterns found in each sample, with higher entropy corresponding to higher diversity (see Materials and methods). We found that although entropy did not correlate with time to relapse (Figure S8 in Additional file 3), the early-divergent samples had, on average, statistically significantly higher entropy than the late-divergent cases at diagnosis (Figure 1E; $P=0.005$, Student's $t$-test; or $P=0.02$, Mann-Whitney test). These analyses indicate that, overall, diagnosis tumors that evolve according to the early-divergent scenario have a more diverse pattern of subclones at diagnosis. This observation is compatible with the observation made above that a minor, highly divergent subclone likely gives rise to the relapse tumor in the early-divergent scenario. It does suggest, however, that in such divergent scenarios, many other divergent clones exist at diagnosis besides the one that gives rise to relapse.

\section{Distinctive genetic evolution patterns of the two relapse scenarios}

To further investigate these evolutionary scenarios, we performed exome sequencing of seven pairs of diagnosis and relapse tumors for which we had sufficient materials (three late-divergent, three early-divergent, and one with a different major VDJ (pair 6); Additional file 2). We achieved $65 \pm 17 \mathrm{X}$ sequencing depth on average and at least 20X depth on $81.7 \pm 7.8 \%$ of the region targeted. We performed single nucleotide variants (SNVs) calling using a previously published procedure [14-16]. We directly compared each relapse sample to its matched diagnosis tumor to identify gained or lost coding region nonsynonynmous SNVs at relapse (SNVs within copy number alteration (CNA) regions were excluded; Figure 2A; Additional file 4). The direct comparison between the matching diagnosis and relapse samples provides several advantages: first, it enabled us to identify genetic alterations only associated with the relapse process of each patient; and second, it allowed us to exclude the majority of the individual specific SNPs (that are not in SNP databases such as dbSNP) without sequencing somatic control DNA, which the historical surgical pathology specimens often lack. Indeed, for three patients for whom there were sufficient amounts of high quality material, we performed exome 


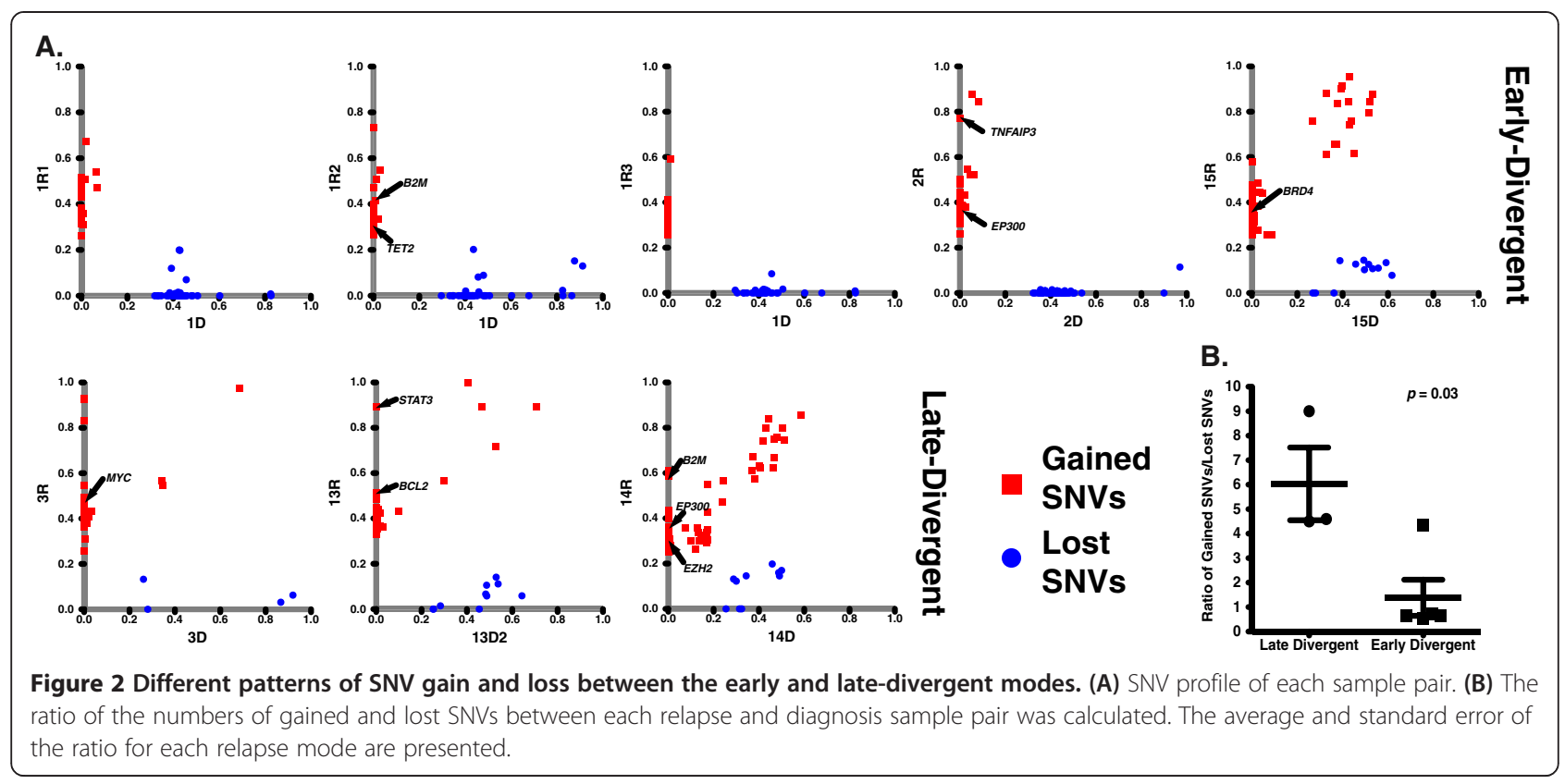

sequencing on somatic control DNA (2C of sample pair 2, 3C of sample pair 3, and $13 \mathrm{C}$ of sample pair 7; Additional file 2), and found that $90.4 \%$ of gained and lost coding region nonsynonymous SNVs identified in their respective tumor samples were not mutated in the somatic control DNA. Of note is that our analysis focused on SNVs whose allelic frequency changes significantly between diagnosis and relapse (in either direction). We used Fisher exact with $10 \%$ false discovery rate control to determine significance. This analysis does not remove variants whose allelic frequency changes significantly between diagnosis and relapse but that are nonetheless present in germline. Since it is not impossible that these variants contribute to some aspects of the disease, we left them in the analysis. In addition, we performed Sanger sequencing on the remaining three sample pairs whose somatic control DNA was in low quantities that it was not suitable for exome study (1C of sample pair $1,2 \mathrm{C}$ of sample pair 2, and $15 \mathrm{C}$ of sample pair 9). We randomly selected $37 \mathrm{SNVs}$ from these three samples to validate and $94.6 \%$ them (35 out of 37 ) were confirmed to be somatic (Additional file 5). These results validate our approach as a robust method to identify specific disease-associated mutations in tumor samples even in the absence of germline controls. We found that overall relapse samples gained coding region nonsynonymous SNVs in 305 genes, among which only 71 were mutated in other previously reported primary DLBCL genomic sequencing studies, including BCL2, EP300, KMT2D, MYC, TET2, and TNFRSF14 [17-20]. We also found one late-divergent mode patient (pair 8, sample ID 14R) with relapse-specific EZH2Y641 mutation (Figure 2A), indicating that relapse
DLBCL can also acquire new EZH2 mutations and that, in addition to its roles in initiating and maintaining DLBCL [21], mutant EZH2 may also contribute to disease relapse. For patient 1, we were able to obtain biopsies from three different relapse sites (Additional file 1). We compared the coding region nonsynonymous SNVs gained in these three relapse samples with the original diagnosis sample and found all of them gained mutations in BET1L, GNAS, and UBR4 genes, suggesting these mutations may be responsible for the initial transformation to relapse disease. Interestingly, relapse tumors $1 \mathrm{R} 2$ and $1 \mathrm{R} 3$ had more overlapping mutations, indicating that these two relapse tumors were more clonally related than they were to $1 R 1$. The numbers of gained and lost SNVs between diagnosis and relapse samples were variable among patients (Additional file 4). On average, the late-divergent relapse samples gained $44.3 \pm 17.6 \mathrm{SNVs}$ and lost $7.5 \pm 1.9$ SNVs compared with their diagnosis samples; in contrast, the early-divergent relapse samples gained $32.0 \pm 5.6$ and lost $35.8 \pm 6.6$ SNVs. The late-divergent scenario relapse samples gained roughly four times more coding region nonsynonymous SNVs than they lost compared with their respective diagnosis samples (5.2 \pm 1.9-fold; Figure 2B), representing the continuous alteration of the tumor genome with additional mutations acquired to achieve relapse. On the contrary, the early-divergent scenario relapse samples gained and lost approximately equal numbers of coding region nonsynonymous SNVs compared with the diagnosis samples $(1.4 \pm 0.7$-fold; Figure $2 \mathrm{~B})$. The coding region nonsynonymous SNV spectrums of the relapse-diagnosis pairs confirm the two tumor evolution modes uncovered by VDJ sequencing. Tumors in 
the early-divergent scenario undergo parallel evolution of their diagnosis and relapse DLBCL genome early during tumor development and therefore acquire new genomic mutations independently and share fewer mutations in common (that is, appear to lose more SNVs). Whereas in the late-divergent scenario, relapse tumors originate from subclones generated during the late stage of tumor evolution and therefore share more mutations in common with the diagnosis tumors (that is, appear to lose fewer SNVs). The presence of 'lost' SNVs in the relapsed tumor compared with the diagnosis tumors within the late-divergent category argues against a 'direct evolution' scenario in which the relapse tumors are generated directly from the major diagnosis subclone. If that had been the case, the relapsed tumors should have shown no lost SNVs.

\section{Immune surveillance genes are specifically targeted by indels and deletions in relapse}

In addition to coding region nonsynonymous SNVs, we identified 39 small indels gained in relapse samples that targeted the coding regions of 36 genes (Additional file 6). On average, each relapse sample gained $5.2 \pm 3.8$ indels. Moreover, we identified CNAs by comparing each relapse exome with its respective diagnosis exome. We identified CNA segments specific to each relapse sample by using a previously widely used and validated $R$ package, DNAcopy $[22,23]$ applied to sequencing depth-adjusted relapse versus diagnosis read count log ratios (Figure S9 in Additional file 3; see Materials and methods). Some CNAs span large chromosome regions, while some are focal alterations (Figure 3; Additional file 7). On average, relapse tumors acquired 55.6 \pm 6.8 CNAs, including $25.0 \pm 5.0$ amplifications and $30.6 \pm 3.5$ deletions. We did not detect CNAs that were common to all the relapse samples. However, we observed several loci that were deleted in multiple relapse samples compared with their respective diagnosis samples (Table 2). We validated two such loci by TaqMan copy number assays, both of which showed loss of genetic material in the relapse samples compared with their respective diagnosis samples (Figure S10 in Additional file 3). These commonly deleted regions harbor genes that are potentially important for B-cell development and malignant transformation (Table 2). For example, three relapse samples (1R3, 3R, 14R) had a relapse-specific deletion spanning $C D 58$, a gene that has been shown to be genetically altered in a subset of DLBCLs [24]. Furthermore, 14R had a relapse specific frameshift indel within the remaining allele of the CD58 gene (Additional file 6). Alteration of this gene may help tumor B cells to escape immune surveillance mechanisms [24]. In addition to CD58 deletion in these three relapse samples, we also found that samples $1 \mathrm{R} 2$ and $14 \mathrm{R}$ gained coding region nonsynonymous SNVs in $B 2 M$ (Additional file 4), another gene that is involved in immune surveillance escape [24]. There was also a relapse-specific frameshift indel within $B 2 M$ in sample 15R (Additional file 6). Overall, in five out of seven patients we sequenced, there were coding region nonsynonymous SNVs, frameshift indels, and gross chromosomal deletions targeting $C D 58$ and $B 2 M$ genes, suggesting that escaping immune surveillance via mutations in key genes, such as $C D 58$ and $B 2 M$, may represent a common relapse strategy. Two other deleted genes, ARHGEF7 and PLCB2, are involved in RAC1 activation and downstream effects [25-27]. Deletions of these two genes may impair RAC1-mediated B-cell receptor signaling [28]. In addition, deletion of $I L 9 R$ may affect JAK-STAT signaling in response to IL9 [29], which is another pathway important in normal B cells and lymphomas [30].

\section{Ultra-deep target resequencing of relapse-specific mutations in diagnostic DLBCL with early-divergent mode of relapse identified minor subclones}

By tracing VDJ SHM, we identified two clonal evolution scenarios of DLBCL relapse. We reasoned that the evolution of genetic events in DLBCL should follow similar patterns. In the case of relapse with an early-divergent mode, it is expected based on VDJ SHM analysis that a small subclone exists within the diagnosis tumor that carries a portion of relapse-specific SNVs or indels, which were not detected by whole exome sequencing but may be identified through deeper sequencing. To test this idea, we performed targeted re-sequencing of a number of SNVs and indels in early-divergent pairs. We obtained roughly two million sequencing reads for each amplicon, which allowed us to detect minor allele frequency of $0.001 \%$ with $20 \mathrm{X}$ sequencing depth. Indeed, targeted re-sequencing revealed that relapse-specific SNVs and indels could be found in a small number of cells at diagnosis in the early-divergent cases. For example, patient 1 gained at relapse a non-synonymous SNV in the UBR4 gene (chr1:19519971, C > A) that produced a premature stop codon. Targeted re-sequencing of this locus confirmed this SNV in the relapse sample of this patient (1R1) (Figure 4A). Interestingly, we observed a very small portion of the tumor cells at diagnosis (1D) also carried this SNV (Figure 4A). This small clone accounted for $0.16 \%$ of the total diagnosis tumor population (background $\mathrm{C}>\mathrm{A}$ conversion within this amplicon was $0.03 \% \pm 0.03 \%)$. In addition, the genetic evolution pattern of this SNV during relapse of patient $1 \mathrm{mir}-$ rored this patient's $\mathrm{V}_{\mathrm{H}} \mathrm{DJ} \mathrm{H}_{\mathrm{H}}$ clonal evolution pattern (Figure 4B). In another example, the relapse tumor of pair 9 (15R) gained a frameshift indel within the $B 2 M$ gene (chr15:45003781-45003782). Target re-sequencing confirmed that $53 \%$ of the relapse sequencing reads carried this specific indel (Figure 4C, 15R, left y-axis), while $0.04 \%$ of the diagnosis population also carried 


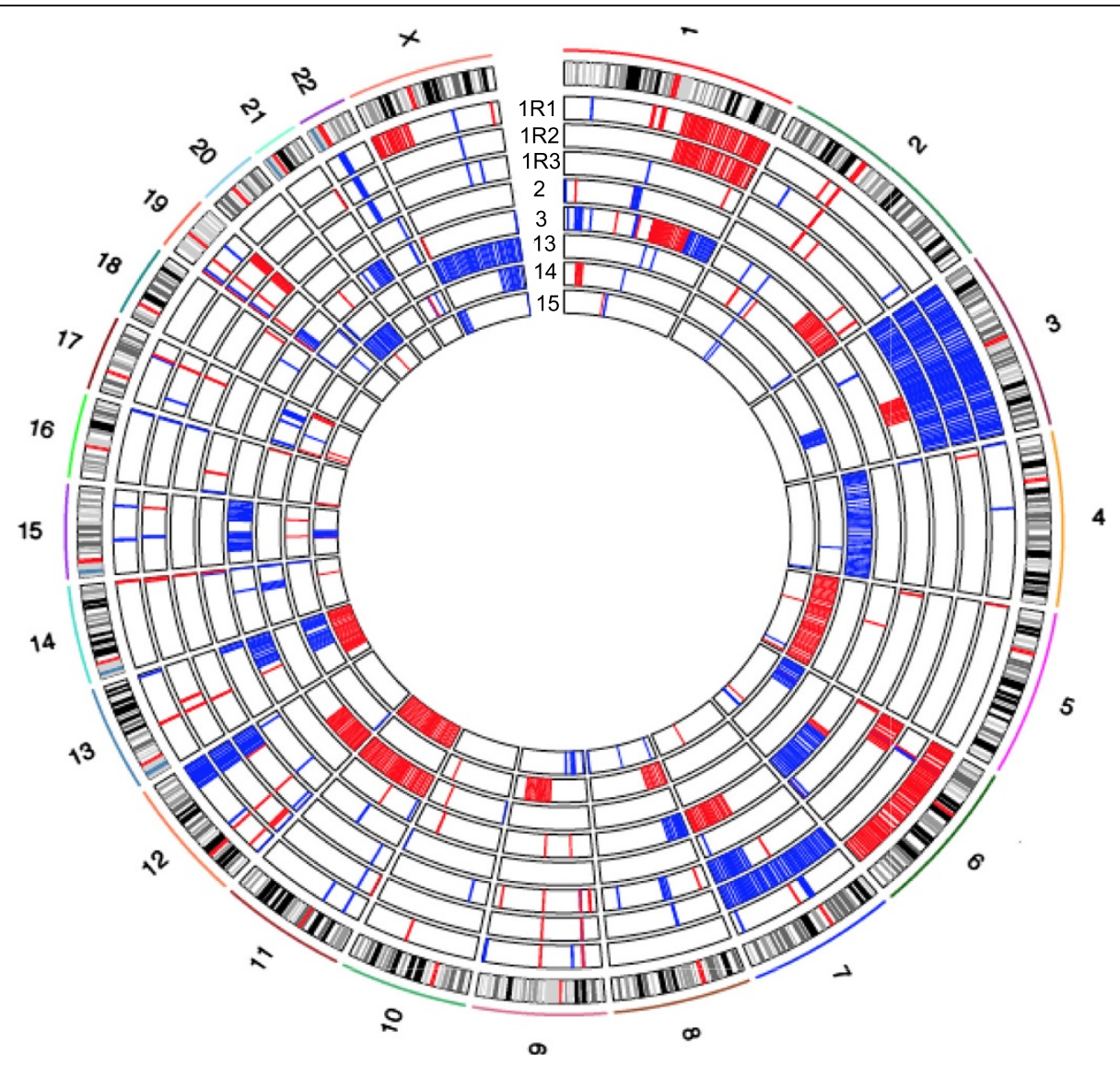

Figure 3 Summary of copy number alteration information. The inner circles represent the CNA of each sample pair. Red indicates copy number gain, and blue indicates copy number loss.

the exact same frameshift indel (Figure 4C, 15D1 and $15 \mathrm{D} 2$, right $\mathrm{y}$-axis; background indel rate $0.004 \pm 0.010 \%$ ). Although we did not observe any subclone in the diagnosis sample carrying the same major relapse $\mathrm{V}_{\mathrm{H}} \mathrm{DJ} \mathrm{H}_{\mathrm{H}}$ sequence, probably due to the difficulty of aligning $\mathrm{V}_{\mathrm{H}} \mathrm{DJ}_{\mathrm{H}}$ sequencing reads (Figure $4 \mathrm{D}$ ), ultra-deep target amplicon re-sequencing allowed us to detect this relapse-causing subclone. Taken together, our results further confirmed the existence of small relapse-causing subclones in the diagnosis tumors of the early-divergent relapse scenario.

\section{Coding-region SNV changes reveal potential mechanisms} of DLBCL relapse

To further explore the potential mechanisms of DLBCL relapse, we performed pathway enrichment analysis [31] on genes where we observed gain of coding region nonsynonymous SNVs in relapse samples (false discovery rate $<10 \%$; Figure 5). Pathway analysis revealed several interesting characteristics of DLBCL relapse. First, relapse samples in both groups gained additional mutations in genes that regulate apoptosis (GO:0006915). Many of these additional mutated genes are pro-apoptotic, such as

Table 2 Summary of common deleted regions in relapse samples

\begin{tabular}{lllll}
\hline Chromosome & $\begin{array}{l}\text { Common deleted } \\
\text { region coordinates }\end{array}$ & Sample ID & Genes & Function in B cells \\
\hline 1 & $117064566-117131520$ & $1 \mathrm{R} 3,3 \mathrm{R}, 14 \mathrm{R}$ & CD58 & Genetically inactivated in DLBCL to escape immune recognition [24] \\
4 & $961371-967030$ & $1 \mathrm{R} 1,2 \mathrm{R}, 13 \mathrm{R}$ & $\mathrm{DGKQ}$ & Not known \\
13 & $111885580-111996434$ & $1 \mathrm{R} 1,2 \mathrm{R}, 3 \mathrm{R}, 14 \mathrm{R}$ & ARHGEF7 & $\begin{array}{l}\text { Guanine nucleotide exchange factor for RAC1 GTPases that } \\
\text { plays an important role in mature B-cell development [25] }\end{array}$ \\
15 & $40594724-40628804$ & $1 \mathrm{R} 1,3 \mathrm{R}, 15 \mathrm{R}$ & PLCB2 & Activated by RAC1 to modulate Ca ${ }^{2+}$ signaling [26] \\
$X$ & $155003966-155250615$ & $3 \mathrm{R}, 13 \mathrm{R}, 15 \mathrm{R}$ & VAMP7, IL9R & IL9R activates JAK-STAT signaling in response to IL9 [29] \\
\hline
\end{tabular}




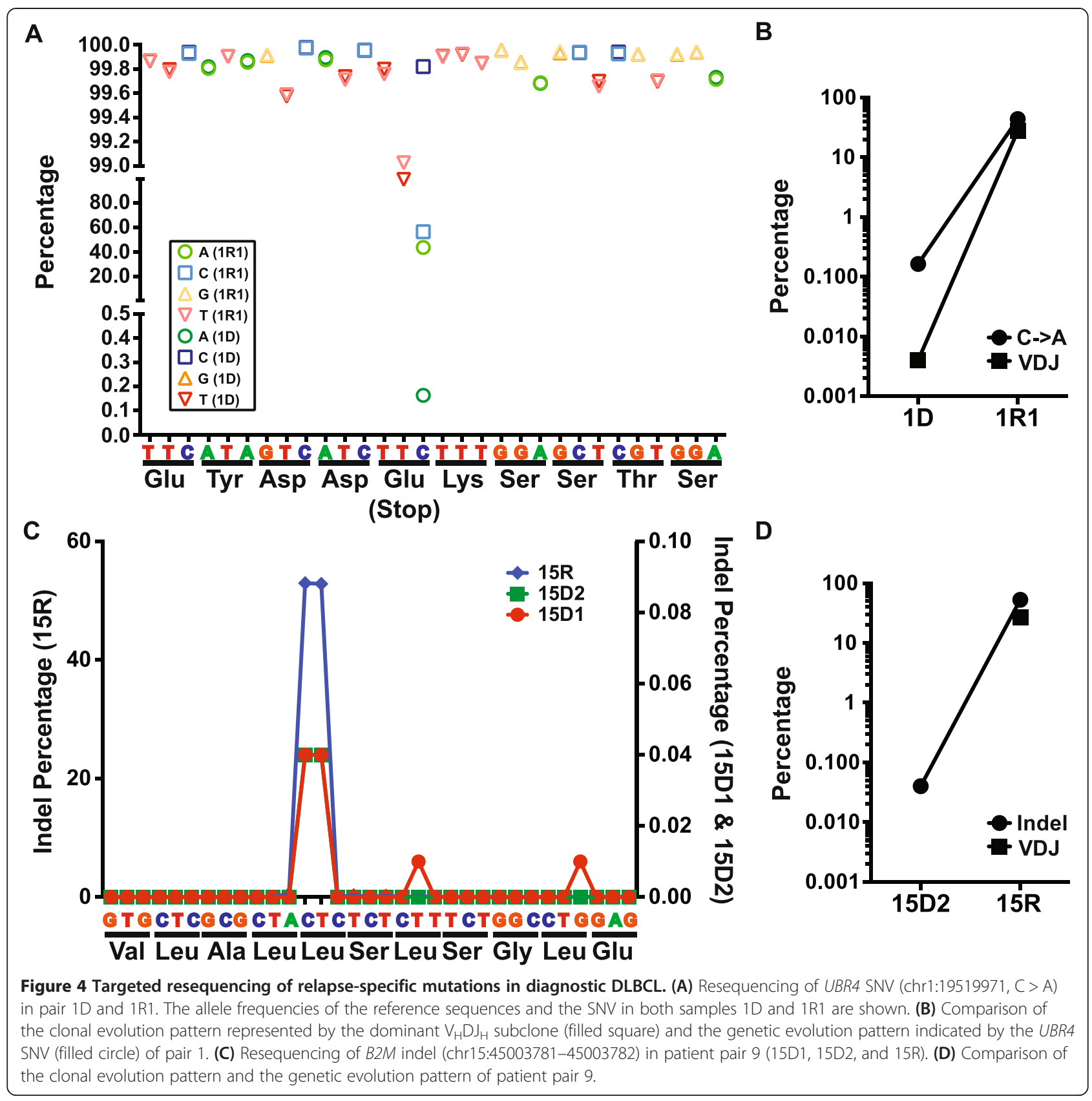

CASP8, BID, and SIAH1, suggesting that for relapse tumors to develop, tumor cells might need to acquire extra survival advantages. Second, relapse samples gained mutations in transmembrane receptor tyrosine kinases (GO:0004714), among which were EPHB2 and $E P H B 6$, family members of the ephrin receptors, which are involved in cell-cell signaling. Third, latedivergent relapse samples gained more mutations in genes that are involved in calcium channel activity (GO:0005262) and p53 binding (GO:0002039). Interestingly, calcium signals play a critical role in B-cell development and functions, and are regulated by many signaling pathways, including B-cell receptor signaling [32]. Our data further suggest that DLBCL tumors with defects in calcium flux regulation may be resistant to current therapy and prone to relapse. Finally, a recent report by Xu-Monette et al. [33] demonstrated that patients with p53 mutations had worse overall and progression-free survival compared with those without. Our data now suggest that DLBCL relapse patients with mutations in p53 functional partners may also have similar outcomes. 


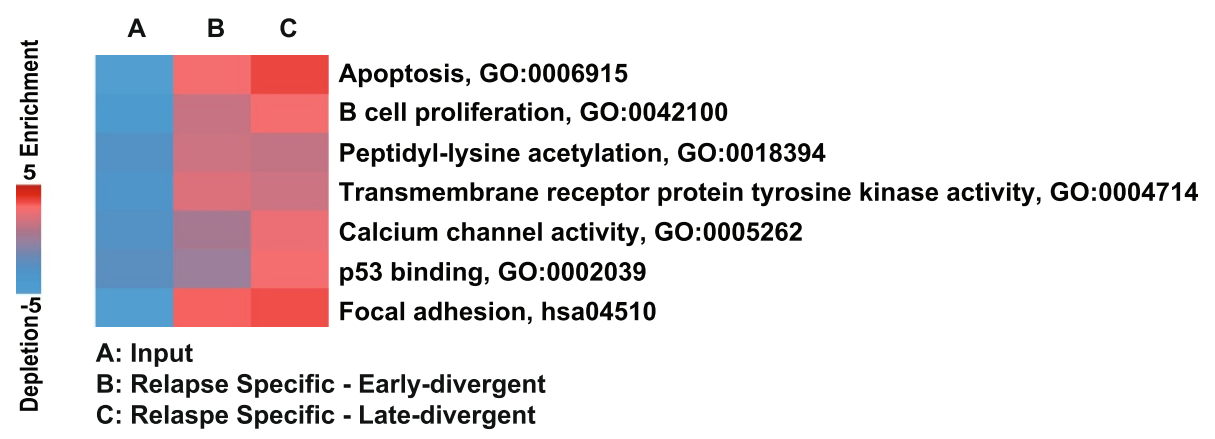

Figure 5 Pathway analysis on genes with coding-region SNVs revealing interesting characteristics of the early- and late-divergent relapse modes. PAGE (Pathway Analysis of Gene Expression) pathway enrichment analysis was performed on gained SNV genes specific to either late-divergent pairs or early-divergent pairs. The enriched functional groups are listed with a heat map representing the degree of enrichment.

\section{Mutations within histone modifiers are potential 'driver' mutations of DLBCL}

Exome sequencing data on paired diagnosis and relapse samples in the early-divergent evolution mode provide us with an opportunity to study the early events in lymphomagenesis. We reasoned that mutations that occurred in both diagnosis and relapse tumors have been acquired early in the life of these tumors and could have acted as early 'driver' or 'facilitator' mutations that initiate tumorigenesis. We observed that in all three divergent pairs, histone-modifying enzymes were mutated in both relapse and diagnosis tumors (Additional file 8). For example, we detected KMT2D and SETDB1 mutations in both relapse and diagnosis tumors of pair 1,KMT2D mutations in pair 2, and EP300 mutations pair 9. The frequencies of all of these SNVs were comparable between the diagnosis and relapse samples within the same pair (Additional file 8). In pair 2, we had limited amounts of germline tissue at our disposal and performed Sanger sequencing to confirm that $K M T 2 D$ mutation is not found in germline DNA (Figure S11 in Additional file 3). These observations suggested that mutations of histone modifiers could act as an early event to establish an aberrant epigenetic landscape in tumor-initiating cells and eventually drive the malignant transformation. Moreover, we observed that relapse samples in the early-divergent group gained additional coding region SNVs in epigenetic modifiers; that is, sample $1 \mathrm{R} 2$ in pair 1 gained a TET2 SNV, sample $2 \mathrm{R}$ of pair 2 gained a SNV in EP300, and sample 15R of pair 9 gained a BRD4 SNV (Additional file 4). These findings suggested that epigenetic modifiers were further targeted during the relapse process, resulting in potential chemoresistance and other features that facilitate the development of relapse disease.

\section{Discussion}

When cancer patients relapse, their tumors often become more aggressive, chemoresistant, and refractory to treatment. The molecular pathogenesis of cancer relapse is largely unknown due to challenges in examining tumor heterogeneity and clonal evolution between diagnosis and relapse tumors in many types of cancers. Here we sought to investigate the molecular mechanisms of DLBCL relapse, utilizing the ability to track tumor heterogeneity and clonal evolution through examination of $\mathrm{V}_{\mathrm{H}} \mathrm{DI} \mathrm{J}_{\mathrm{H}}$ rearrangements and SHM, as well as exome sequencing. To exhaustively catalog the $\mathrm{V}_{\mathrm{H}} \mathrm{DJ} \mathrm{J}_{\mathrm{H}}$ repertoires of the tumor samples, we adapted next-generation sequencing technology to sequence the IgVDJ rearrangements in great depth. This approach has been successfully used in other studies to examine human antibody repertoires [12,34,35], assess clonal heterogeneity in B-ALL or chronic lymphocytic leukemia [10,36,37], or monitor minimal residual disease in chronic lymphocytic leukemia [38]. We observed a wide range of VDJ heterogeneity within diagnosis DLBCL samples, but no significant differences in this between the paired diagnosis and relapse tumors, suggesting that tumor heterogeneity was preserved after disease progression due to either the incomplete eradication of the diagnosis tumor or ongoing VDJ rearrangement during relapse. However, we could not completely rule out the possibility of nontumor B-cell contamination.

Because DLBCL is a clonal disease, we decided to focus on the major $\mathrm{V}_{\mathrm{H}} \mathrm{DJ} \mathrm{H}_{\mathrm{H}}$ rearrangement clone. We found that, with one exception, the majority of the diagnosis and tumor pairs had the same major $\mathrm{V}_{\mathrm{H}} \mathrm{DJ}_{\mathrm{H}}$ rearrangement, indicating they were clonally related, even when the relapse tumors developed more than 5 years after the diagnosis tumor, similar to what has been reported before [39]. We identified two distinct patterns of SHM amongst the diagnosis-relapse sample pairs, suggesting two modes of relapse disease development. In addition, exome sequencing on several sample pairs with different SHM patterns also revealed profound evidence to further support the idea that there are two discrete pathogenesis mechanisms of DLBCL relapse. In the late-divergent mode, the diagnosis-relapse pairs exhibited: 1) almost identical SHM frequency and distribution of the subclones that they clustered together in the phylogenetic tree; 2) highly 
overlapping SNV profiles with additional gained SNVs in the relapse samples; 3 ) ongoing genomic instability that resulted in CNA in relapse samples. In the earlydivergent mode, the diagnosis-relapse pairs exhibited: 1) vastly different distribution of SHM, which led to segregation in the phylogenetic tree; and 2) less overlapping SNV profiles in which relapse tumors 'gained' and 'lost' similar numbers of SNVs compared with diagnosis tumors. Taken together, our results suggest that DLBCL relapse develops either from a late subclone within the diagnosis tumor, which shares the majority of the mutations with the major diagnosis clone (late-divergent mode), or from a highly diverged, relatively early minor subclone within the diagnosis tumor that may share some tumor-initiating (driver) mutations with the major diagnosis clone but may acquire additional independent facilitating (facilitator) mutations important for lymphomagenesis at a later time point during relapse (early-divergent mode) (Figure 6). Indeed, by performing ultra-deep targeted re-sequencing of SNVs and indels gained in relapse samples, we confirmed in the diagnostic tumors the presence of minor subclones that already carry some of the same mutations as the relapse tumors in the early divergent scenario. The frequency of the diagnosis tumor cells harboring relapsespecific SNVs and indels was similar to the frequency of diagnosis cells carrying the same major relapse $\mathrm{V}_{\mathrm{H}} \mathrm{DJ}_{\mathrm{H}}$ sequences, implying that these subclones indeed are the precursors of the final relapse tumors. Taken together, our data suggest that a small subpopulation of the lymphoma cells may survive chemotherapy, possibly due to acquisition of additional mutations that confer chemotherapy resistance or 'hiding' of the tumor cells in protective cellular milieu (for example, BM). This subclone may then acquire additional facilitator mutations important for full generation of relapse at a later time point. A similar scenario is likely to operate in the late-divergent scenario. Ultra-deep targeted re-sequencing of relapse-specific alterations will be useful to further investigate the size of relapse precursor subclones present in the diagnostic tumors in those cases. Recently, Pasqualucci et al. [40] demonstrated that follicular lymphomas (FLs) and transformed FLs evolve via linear or divergent evolution patterns by examining FL exomes. By characterizing both the immunoglobulin gene and the rest of the genome, we showed similar evolution patterns for DLBCL relapse, suggesting that these evolution patterns may be common to lymphoid malignancies.

In recent years, several studies have examined primary (non-relapse) DLBCL exomes and discovered many recurrent mutations [17-20,41]. However, with one exception, that is, EZH2 [21], the lack of knowledge about how these mutations lead to disease development hinders the development of effective targeted therapies. To date, it is still unclear whether these mutations are 'driver' mutations that initiate the malignant transformation, 'facilitator' mutations that promote disease progression, or just merely 'passenger' mutations that have no effects on the disease pathogenesis. The early-divergent mode of tumor evolution uncovered here provided us with an opportunity to identify early driver mutations in lymphomagenesis via 'ancestral' tumor reconstruction. Indeed, common mutations between the diagnosis and relapse tumors are likely to have been acquired early and constitute potentially disease-initiating mutations that are acquired by the tumors at the earliest stage. By comparing and contrasting exomes of diagnosis and relapse samples, we found that epigenetic modifiers, such as EP300, KMT2D, and SETDB1, were mutated in both diagnosis and relapse tumors, suggesting that these mutations could be the driver mutations of DLBCL. Similarly, Green et al. [42] also reported that $C R E B B P$ mutations were early 'driver' mutations in FL because they were found in the CD20 subpopulations in both diagnosis and relapse FLs, while KMT2D mutations were likely later 'accelerators' in FL since they were only found in one of the subpopulations. Moreover, KMT2D, CREBBP, and EP300 are among the most frequently mutated genes in DLBCL and FL [17-20,41]. Therefore, we hypothesize that mutations within epigenetic modifiers may act as early driver events in lymphomagenesis and help establish an aberrant epigenetic environment suitable for subsequent malignant transformation. Further experiments modeling these mutations are needed to elucidate their functions in driving lymphomagenesis. In addition, we observed relapse samples of the early-divergent mode gained extra mutations in epigenetic modifiers. A similar phenomenon has also been observed in other relapse diseases, such as CREBBP and SETD2 mutations enriched in relapse B-ALL $[43,44]$. All together, these observations suggest that mutations in epigenetic modifiers may be responsible for the chemoresistance feature of these tumors allowing them to survive or escape initial therapies and develop into relapse tumors. Moreover, through our mutation analysis, we identified several pathways that may be involved in the relapse process. These pathways include apoptosis regulation, transmembrane receptor tyrosine kinases, calcium channel activity and p53 binding. Several these pathway genes and their family members have been implicated in lymphomagenesis previously. For example, relapse samples gained mutations in transmembrance receptor tyrosine kinase genes $E P H B 2$ and $E P H B 6$, whose family member EPHA7 encodes a known soluble tumor suppressor for FL [45]. Our results suggest that this family of tyrosine kinases may play an additional role in preventing DLBCL relapse. Moreover, because of the importance of calcium-dependent regulation of NFAT and NF- $\mathrm{kB}$ activities in the determination of cell-fate choice of $B$ cells during humoral immune responses, and the chronic activation of B-cell receptor signaling and elevated calcium signaling in aggressive 


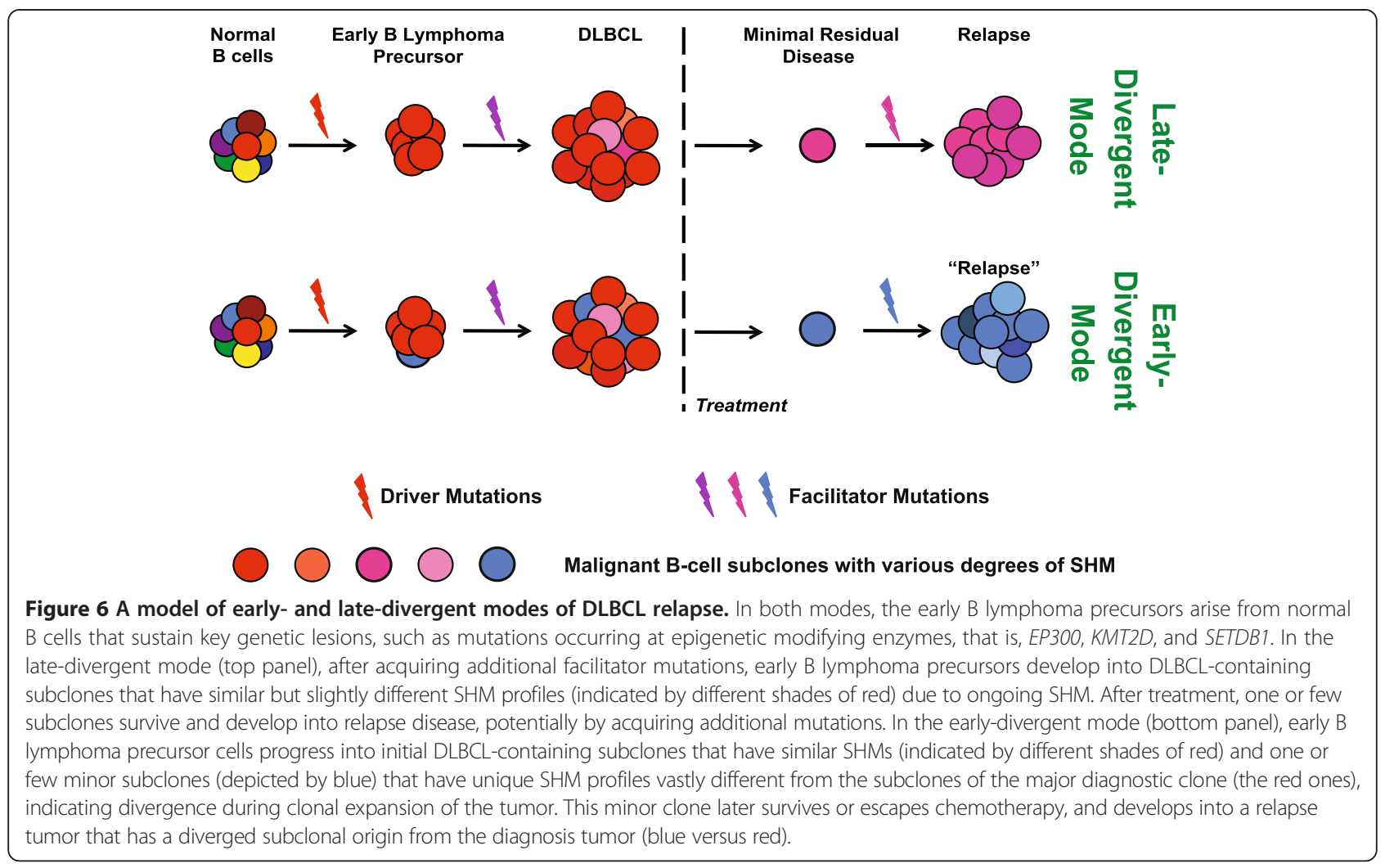

Activated B-cell-like DLBCL [46], it would be interesting to further investigate the role of this pathway in the DLBCL relapse process and develop targeted therapy against this pathway to treat relapse DLBCL. Indeed, targeting B-cell receptor signaling components has already been shown to be beneficial for relapsed/refractory B-cell malignancies [47].

\section{Conclusions}

Using ultra-deep sequencing of the rearranged IgH locus and the exomes of diagnosis and relapse DLBCL tumor pairs, we identified two distinct evolutionary scenarios that lead to relapse. In one scenario, the relapse clone evolves directly from the main diagnosis clone via the acquisition of additional relapse-driving mutations. We termed this scenario the 'late-divergent' mode. In the other scenario, diagnosis and relapse evolve in parallel from a common, early progenitor cell and carry very different patterns of somatic mutations. We named this scenario the 'early-divergent' mode. Our data further suggest that mutations within epigenetic modifiers could occur early in lymphomagenesis and act as the driving events. We also identified frequent genetic alterations in immune surveillance genes (B2M and CD58), suggesting immune escape contributes to lymphoma relapse. Therefore, our study presents important evidence for the first time that DLBCL relapse may result from multiple different pathogenesis mechanisms, providing rationales for the design of distinct therapies for each mechanism. Moreover, this study highlights the urgent need for understanding the roles of epigenetic modifier and immune escape mutations in driving lymphomagenesis and the relapse phenotype.

\section{Materials and methods Case selection}

Diagnosis and relapse DLBCL cases were selected from a search of the database of the Department of Pathology and Laboratory Medicine at Weill Cornell Medical College and Department of Pathology at Singapore General Hospital. Information regarding clinical history and presentation, therapy and follow-up was obtained from electronic clinical records. All patients provided written consent for use of tissues samples for research, in accordance with the Declaration of Helsinki regulations of the protocols approved by the Institutional Review Board of Weill Cornell Medical College, New York, USA (IRB \# 0107004999), and by SingHealth Services, Singapore (IRB \# 2006/036/B).

\section{DNA extraction}

DNA was extracted from either frozen solid tissue sections or formalin-fixed paraffin-embedded tissue sections. Frozen tissue samples were first digested overnight with $0.5 \mathrm{mg} / \mathrm{ml}$ Proteinase $\mathrm{K}$ and $0.625 \%$ SDS in $4 \mathrm{ml}$ nucleic lysis buffer at $37^{\circ} \mathrm{C}$. After digestion, $1 \mathrm{ml}$ of saturated $\mathrm{NaCl}$ was added to the samples and samples were 
shaken vigorously for $15 \mathrm{~s}$ before being spun at 2,500 rpm for 15 minutes. Supernatant was transferred to a new tube and mixed with two volumes of room temperature $100 \%$ ethanol. DNA was precipitated by centrifugation at maximum speed for 30 minutes, washed twice with $70 \%$ ethanol, and finally dissolved in TE or nuclease-free water overnight at room temperature. Formalin-fixed paraffinembedded samples were de-paraffined first by incubating in Xylene at room temperature for 10 minutes twice followed by incubating with $100 \%$ ethanol at room temperature for 10 minutes twice. Samples were then allowed to air-dry and then incubated with $0.5 \mathrm{mg} / \mathrm{ml}$ Proteinase $\mathrm{K}$ in $1 \mathrm{X}$ PCR buffer overnight at $37^{\circ} \mathrm{C}$ followed by $95^{\circ} \mathrm{C}$ for 10 minutes to heat inactivate Proteinase $\mathrm{K}$.

\section{$\mathrm{V}_{\mathrm{H}} \mathrm{DJ} \mathrm{J}_{\mathrm{H}}$ sequencing}

The IgVHFR1 VDJ junctions were amplified using mix 2 of the Somatic Hypermutation Assay from InvivoScribe Technologies (San Diego, CA, U.S.A.). The IgVHFR2 VDJ junctions were amplified using Tube B of IGH Gene Clonality Assay from InvivoScribe Technologies. PCR products were resolved on $2 \%$ agarose gel and the major product of the appropriate size was purified by using a QIAGEN MiniElute Gel Extraction kit (Valencia, CA, U.S.A.). Sequencing libraries were constructed from the purified PCR product by using Illumina TruSeq DNA Sample Preparation Kit v2 (San Diego, CA, U.S.A.). Each sample was tagged with a unique index. For each MiSeq sequencing run, five VDJ samples were mixed together at $7 \mu \mathrm{M}$ with $50 \%$ PhiX spike-in to ensure the complexity of the run.

\section{Exome sequencing}

Exome sequencing samples were prepared using the Aglient SureSelect ${ }^{\mathrm{XT}}$ Human All Exon 50 MB Target Enrichment System for Illumina Paired-End Sequencing Library kit. PE75 sequencing was performed on Illumina HiSeq 2000. For sample 1, we sequenced all three relapse samples that were obtained at three separate biopsy sites, and analyzed them independently.

\section{$\mathrm{V}_{\mathrm{H}} \mathrm{DJ} \mathrm{J}_{\mathrm{H}}$ analysis pipeline}

Paired-end sequence reads were mapped against a human IGH reference database available from the IMGT website [9] using a modified nucleotide blast search. Sequences without a hit in all three V, D and J regions were filtered out and reads with all three regions present were counted for the number of each unique rearrangement. These counts were then ranked for each sample and the major rearrangements were then aligned against their corresponding dominant $\mathrm{V}_{\mathrm{H}} \mathrm{DJ}_{\mathrm{H}}$ sequences. To perform phylogenetic analysis, within each sample we selected the subclones of the major $\mathrm{V}_{\mathrm{H}} \mathrm{DJ} \mathrm{H}_{\mathrm{H}}$ rearrangement that had at least 10 sequencing counts and a minimum $80 \%$ sequence similarity to the reference germline VDJ sequence. We then ranked these subclones in the diagnosis sample based on their similarity to the SHM profile of the respective relapse sample, and vice versa. The top 10 ranked subclones along with the 10 most abundant clones and a random selection of minor subclones from each diagnosis and relapse samples within each pair were then re-aligned using the multiple sequence alignment tool Clustalw to build a neighbor-joining tree by using the $\mathrm{R}$ package 'ape' [13]. The tree coordinates alongside the corresponding frequencies for each alignment were drawn using a custom script.

\section{Exome analysis pipeline SNV discovery}

Short sequencing reads were aligned to human genome assembly GRCh37/hg19 using the BWA aligner [48]. Duplicated paired reads were filtered and variant detection was performed as previously described [14-16]. Novel coding region SNVs (not present in SNP132) were further filtered according to sequencing depth $(\geq 20 \mathrm{X})$ and variant percentage $(\geq 25 \%)$. To analyze the mutational status change (gain or loss) within each diagnosis and relapse pair, we compared the variant ratio of each novel coding SNV between the diagnosis and relapse samples and estimated the statistical significance of the difference by using a Chi-square tested corrected with multiple hypothesis testing (Benjamini-Hochberg corrected $P<0.1$ ). To obtain the list of common novel coding region SNVs, we took those SNVs that did not show a significant different variant ratio between the diagnosis and relapse samples (corrected $P>0.1$ ) and selected the ones that were mutated in at least two non-Hodgkin's lymphoma patients in other studies.

\section{CNA segment calling}

In order to call CNA segments, aligned short sequencing reads were used to generate $\log 2$ ratios between the diagnosis and relapse samples for each patient using an in-house program, CNVseeqer. The log2 ratios represent the number of reads mapped to an exon in the relapse sample compared with a diagnosis sample. Log2 ratios of exons that had more than 100 total reads between both the diagnosis and relapse samples were smoothed and then used for segmentation identification using a circular binary segmentation algorithm DNAcopy in R [22]. Segments with a standard deviation $>1.5$ were defined as CNA segments. Segments with a mean $\log 2$ ratio $>0.3$ were categorized as copy number gain loci, while segments with a mean $\log 2$ ratio $<-0.3$ were categorized as copy number loss loci.

\section{Data deposition}

All exome-sequencing raw data files have been deposited into NCBI database BioProject PRJNA240335 [49]. 
All targeted sequencing raw data files, including VDJsequencing and targeted resequencing of the UBR4 and $B 2 M$ loci, have been deposited into NBCI database BioProject PRJNA240336 [50].

\section{Additional files}

Additional file 1: Table S1. Clinical information of the patient samples used in this study.

Additional file 2: Table S2. Sequencing run metrics. Additional file 3: Supplementary figures.

Additional file 4: Table S3. Coding region nonsynonymous SNVS gained or lost in relapse samples.

Additional file 5: Table S4. Sanger validation results on selected nonsynonymous SNVs gained in relapse samples.

Additional file 6: Table S5. Coding region indels gained in relapse samples.

Additional file 7: Table S6. Gained copy number alterations in relapse samples.

Additional file 8: Table S7. Sequencing results of epigenetic modifier mutations in sample pairs 1, 2, and 9.

\section{Abbreviations}

B-All: acute B lymphoblastic leukemia; BM: bone marrow; bp: base pair; CNA: copy number alteration; DLBCL: diffuse large B-cell lymphoma; FL: follicular lymphoma; GC: germinal center; IGH: immunoglobulin heavy chain; IL: interleukin; PCR: polymerase chain reaction; SHM: somatic hypermutation; SNP: single nucleotide polymorphism; SNV: single nucleotide variant.

\section{Competing interests}

The authors declare that they have no competing financial interests.

\section{Authors' contributions}

YJ, WT, and OE conceived the study. TC, PM, LHCT, and WT collected samples. YJ and KN performed the experiments. YJ, DR, and KWE performed data analyses. YJ, AMM, WT, and OE wrote the manuscript. All authors have read and approved the manuscript. WT and OE are co-senior authors.

\section{Acknowledgements}

We would like to thank Dr Rita Shaknovich and members of Melnick lab, Tam lab, and Elemento lab for thoughtful discussions. We would like to thank the Genomics Resources Core Facility at Weill Cornell Medical College for performing the VDJ and exome sequencing. $\mathrm{YJ}$ is supported by ASH Scholar Award. WT and OE are supported by Weill Cornell Cancer Center Pilot Grant. OE is supported by the NSF CAREER award, the Starr Cancer Consortium and the Hirschl Trust. We would also like to thank Katherine Benesch, JD, MPH for her generous support to this project.

\section{Author details \\ ${ }^{1}$ Institute for Computational Biomedicine, Weill Cornell Medical College, New York, NY 10021, USA. ²Department of Medicine, Weill Cornell Medical College, New York, NY 10021, USA. ${ }^{3}$ Department of Pathology and Laboratory Medicine, Weill Cornell Medical College, New York, NY 10021, USA. ${ }^{4}$ Hematology, Lymphoid Unit, Hôpital Henri Mondor, Creteil 94010, France. ${ }^{5}$ Department of Pathology, Singapore General Hospital, Singapore 169608, Singapore.}

Received: 22 July 2014 Accepted: 7 August 2014 Published: 15 August 2014

\section{References}

1. Coiffier B, Lepage E, Briere J, Herbrecht R, Tilly H, Bouabdallah R, Morel P, Van Den Neste E, Salles G, Gaulard P, Reyes F, Lederlin P, Gisselbrecht C: CHOP chemotherapy plus rituximab compared with $\mathrm{CHOP}$ alone in elderly patients with diffuse large-B-cell lymphoma. N Engl J Med 2002, 346:235-242.
2. Larouche JF, Berger F, Chassagne-Clement C, Ffrench M, Callet-Bauchu E, Sebban C, Ghesquieres H, Broussais-Guillaumot F, Salles G, Coiffier B: Lymphoma recurrence 5 years or later following diffuse large B-cell lymphoma: clinical characteristics and outcome. J Clin Oncol 2010, 28:2094-2100.

3. Gisselbrecht C, Glass B, Mounier N, Singh Gill D, Linch DC, Trneny M, Bosly A, Ketterer N, Shpilberg O, Hagberg H, Ma D, Briere J, Moskowitz CH, Schmitz N: Salvage regimens with autologous transplantation for relapsed large B-cell lymphoma in the rituximab era. J Clin Oncol 2010, 28:4184-4190.

4. Friedberg JW: Relapsed/refractory diffuse large B-cell lymphoma. Hematology Am Soc Hematol Educ Program 2011, 2011:498-505.

5. Ding L, Ley TJ, Larson DE, Miller CA, Koboldt DC, Welch JS, Ritchey JK, Young MA, Lamprecht T, McLellan MD, McMichael JF, Wallis JW, Lu C, Shen D, Harris CC, Dooling DJ, Fulton RS, Fulton LL, Chen K, Schmidt H, Kalicki-Veizer J, Magrini VJ, Cook L, McGrath SD, Vickery TL, Wendl MC, Heath S, Watson MA, Link DC, Tomasson MH, et al: Clonal evolution in relapsed acute myeloid leukaemia revealed by whole-genome sequencing. Nature 2012, 481:506-510.

6. Jung D, Alt FW: Unraveling V(D)J recombination; insights into gene regulation. Cell 2004, 116:299-311.

7. Berek C, Berger A, Apel M: Maturation of the immune response in germinal centers. Cell 1991, 67:1121-1129.

8. Jacob J, Kelsoe G, Rajewsky K, Weiss U: Intraclonal generation of antibody mutants in germinal centres. Nature 1991, 354:389-392.

9. Lefranc MP, Giudicelli V, Ginestoux C, Jabado-Michaloud J, Folch G, Bellahcene F Wu Y, Gemrot E, Brochet X, Lane J, Regnier L, Ehrenmann F, Lefranc G, Duroux P: IMGT, the international ImMunoGeneTics information system. Nucleic Acids Res 2009, 37:D1006-D1012.

10. Gawad C, Pepin F, Carlton VE, Klinger M, Logan AC, Miklos DB, Faham M, Dahl G, Lacayo N: Massive evolution of the immunoglobulin heavy chain locus in children with B precursor acute lymphoblastic leukemia. Blood 2012, 120:4407-4417.

11. Yancopoulos GD, Alt FW: Regulation of the assembly and expression of variable-region genes. Annu Rev Immunol 1986, 4:339-368.

12. Arnaout R, Lee W, Cahill P, Honan T, Sparrow T, Weiand M, Nusbaum C, Rajewsky K, Koralov SB: High-resolution description of antibody heavy-chain repertoires in humans. PLoS One 2011, 6:e22365.

13. Saitou N, Nei M: The neighbor-joining method: a new method for reconstructing phylogenetic trees. Mol Biol Evol 1987, 4:406-425.

14. Jiang $Y$, Soong TD, Wang L, Melnick AM, Elemento O: Genome-wide detection of genes targeted by non-lg somatic hypermutation in lymphoma. PLOS One 2012, 7:e40332.

15. Wacker SA, Houghtaling BR, Elemento O, Kapoor TM: Using transcriptome sequencing to identify mechanisms of drug action and resistance. Nat Chem Biol 2012, 8:235-237.

16. Rajadhyaksha AM, Elemento O, Puffenberger EG, Schierberl KC, Xiang JZ, Putorti ML, Berciano J, Poulin C, Brais B, Michaelides M, Weleber RG, Higgins $\mathrm{JJ}$ : Mutations in FLVCR1 cause posterior column ataxia and retinitis pigmentosa. Am J Hum Genet 2010, 87:643-654.

17. Lohr JG, Stojanov P, Lawrence MS, Auclair D, Chapuy B, Sougnez C, Cruz-Gordillo P, Knoechel B, Asmann YW, Slager SL, Novak AJ, Dogan A, Ansell SM, Link BK, Zou L, Gould J, Saksena G, Stransky N, Rangel-Escareno C, Fernandez-Lopez JC, Hidalgo-Miranda A, Melendez-Zajgla J, Hernandez-Lemus E, Schwarz-Cruz y Celis A, Imaz-Rosshandler I, Ojesina Al, Jung J, Pedamallu CS, Lander ES, Habermann TM, et al: Discovery and prioritization of somatic mutations in diffuse large $B$-cell lymphoma (DLBCL) by whole-exome sequencing. Proc Natl Acad Sci U S A 2012, 109:3879-3884.

18. Pasqualucci L, Trifonov V, Fabbri G, Ma J, Rossi D, Chiarenza A, Wells VA, Grunn A, Messina M, Elliot O, Chan J, Bhagat G, Chadburn A, Gaidano G, Mullighan CG, Rabadan R, Dalla-Favera R: Analysis of the coding genome of diffuse large B-cell lymphoma. Nat Genet 2011, 43:830-837.

19. Morin RD, Mendez-Lago M, Mungall AJ, Goya R, Mungall KL, Corbett RD, Johnson NA, Severson TM, Chiu R, Field M, Jackman S, Krzywinski M, Scott DW, Trinh DL, Tamura-Wells J, Li S, Firme MR, Rogic S, Griffith M, Chan S, Yakovenko O, Meyer IM, Zhao EY, Smailus D, Moksa M, Chittaranjan S, Rimsza L, Brooks-Wilson A, Spinelli JJ, Ben-Neriah S, et al: Frequent mutation of histonemodifying genes in non-Hodgkin lymphoma. Nature 2011, 476:298-303.

20. Zhang J, Grubor V, Love CL, Banerjee A, Richards KL, Mieczkowski PA, Dunphy C, Choi W, Au WY, Srivastava G, Lugar PL, Rizzieri DA, Lagoo AS, Bernal-Mizrachi L, Mann KP, Flowers C, Naresh K, Evens A, Gordon LI, Czader M, Gill Jl, Hsi ED, Liu Q, Fan A, Walsh K, Jima D, Smith LL, Johnson AJ, Byrd JC, Luftig MA, et al: Genetic heterogeneity of diffuse large B-cell lymphoma. Proc Natl Acad Sci U S A 2013, 110:1398-1403. 
21. Beguelin W, Popovic R, Teater $M$, Jiang $Y$, Bunting $K L$, Rosen $M$, Shen $H$, Yang SN, Wang L, Ezponda T, Martinez-Garcia E, Zhang H, Zheng Y, Verma SK McCabe MT, Ott HM, Van Aller GS, Kruger RG, Liu Y, McHugh CF, Scott DW, Chung YR, Kelleher N, Shaknovich R, Creasy CL, Gascoyne RD, Wong KK, Cerchietti L, Levine RL, Abdel-Wahab O, et al: EZH2 is required for germinal center formation and somatic EZH2 mutations promote lymphoid transformation. Cancer Cell 2013, 23:677-692.

22. Olshen $A B$, Venkatraman $E S$, Lucito $R$, Wigler M: Circular binary segmentation for the analysis of array-based DNA copy number data. Biostatistics 2004, 5:557-572.

23. Lonigro RJ, Grasso CS, Robinson DR, Jing X, Wu YM, Cao X, Quist MJ, Tomlins SA, Pienta KJ, Chinnaiyan AM: Detection of somatic copy number alterations in cancer using targeted exome capture sequencing. Neoplasia 2011, 13:1019-1025.

24. Challa-Malladi M, Lieu YK, Califano O, Holmes AB, Bhagat G, Murty W, Dominguez-Sola D, Pasqualucci L, Dalla-Favera R: Combined genetic inactivation of beta2-Microglobulin and CD58 reveals frequent escape from immune recognition in diffuse large B cell lymphoma. Cancer Cell 2011, 20:728-740.

25. Manser E, Loo TH, Koh CG, Zhao ZS, Chen XQ, Tan L, Tan I, Leung T, Lim L: PAK kinases are directly coupled to the PIX family of nucleotide exchange factors. Mol Cell 1998, 1:183-192.

26. Harden TK, Hicks SN, Sondek J: Phospholipase C isozymes as effectors of Ras superfamily GTPases. J Lipid Res 2009, 50:S243-S248.

27. Dumont C, Henderson R, Tybulewicz VL: Characterization of the roles of Rac1 and Rac2 GTPases in lymphocyte development. Methods Enzymol 2008, 439:235-254

28. Johmura S, Oh-hora M, Inabe K, Nishikawa Y, Hayashi K, Vigorito E, Kitamura D, Turner M, Shingu K, Hikida M, Kurosaki T: Regulation of Vav localization in membrane rafts by adaptor molecules Grb2 and BLNK. Immunity 2003, 18:777-787

29. Demoulin JB, Uyttenhove C, Van Roost E, DeLestre B, Donckers D, Van Snick J, Renauld JC: A single tyrosine of the interleukin-9 (IL-9) receptor is required for STAT activation, antiapoptotic activity, and growth regulation by IL-9. Mol Cell Biol 1996, 16:4710-4716

30. O'Shea JJ, Holland SM, Staudt LM: JAKs and STATs in immunity, immunodeficiency, and cancer. N Engl J Med 2013, 368:161-170

31. Goodarzi H, Elemento O, Tavazoie S: Revealing global regulatory perturbations across human cancers. Mol Cell 2009, 36:900-911.

32. Young RM, Staudt LM: Targeting pathological B cell receptor signalling in lymphoid malignancies. Nat Rev Drug Discov 2013, 12:229-243.

33. Xu-Monette ZY, Wu L, Visco C, Tai YC, Tzankov A, Liu WM, Montes-Moreno S, Dybkaer K, Chiu A, Orazi A, Zu Y, Bhagat G, Richards KL, Hsi ED, Zhao XF, Choi WW, Zhao X, van Krieken JH, Huang Q, Huh J, Ai W, Ponzoni M, Ferreri AJ, Zhou F, Kahl BS, Winter JN, Xu W, Li J, Go RS, Li Y, et al: Mutational profile and prognostic significance of TP53 in diffuse large B-cell lymphoma patients treated with R-CHOP: report from an International DLBCL Rituximab-CHOP Consortium Program Study. Blood 2012, 120:3986-3996.

34. Freeman JD, Warren RL, Webb JR, Nelson BH, Holt RA: Profiling the T-cell receptor beta-chain repertoire by massively parallel sequencing. Genome Res 2009, 19:1817-1824.

35. Boyd SD, Gaeta BA, Jackson KJ, Fire AZ, Marshall EL, Merker JD, Maniar JM, Zhang LN, Sahaf B, Jones CD, Simen BB, Hanczaruk B, Nguyen KD, Nadeau KC, Egholm M, Miklos DB, Zehnder JL, Collins AM: Individual variation in the germline lg gene repertoire inferred from variable region gene rearrangements. J Immunol 2010, 184:6986-6992.

36. Campbell PJ, Pleasance ED, Stephens PJ, Dicks E, Rance R, Goodhead I, Follows GA, Green AR, Futreal PA, Stratton MR: Subclonal phylogenetic structures in cancer revealed by ultra-deep sequencing. Proc Natl Acad Sci U S A 2008, 105:13081-13086.

37. Boyd SD, Marshall EL, Merker JD, Maniar JM, Zhang LN, Sahaf B, Jones CD, Simen BB, Hanczaruk B, Nguyen KD, Nadeau KC, Egholm M, Miklos DB, Zehnder $J$, Fire AZ: Measurement and clinical monitoring of human lymphocyte clonality by massively parallel VDJ pyrosequencing. Sci Trans/ Med 2009, 1:12ra23.

38. Logan AC, Gao H, Wang C, Sahaf B, Jones CD, Marshall EL, Buno I, Armstrong R, Fire AZ, Weinberg Kl, Mindrinos M, Zehnder JL, Boyd SD, Xiao W, Davis RW, Miklos DB: High-throughput VDJ sequencing for quantification of minimal residual disease in chronic lymphocytic leukemia and immune reconstitution assessment. Proc Natl Acad Sci U S A 2011, 108:21194-21199.
39. de Jong $D$, Glas AM, Boerrigter $L$, Hermus MC, Dalesio O, Willemse $E_{\text {, }}$ Nederlof PM, Kersten MJ: Very late relapse in diffuse large B-cell lymphoma represents clonally related disease and is marked by germinal center cell features. Blood 2003, 102:324-327.

40. Pasqualucci L, Khiabanian H, Fangazio M, Vasishtha M, Messina M, Holmes AB, Ouillette P, Trifonov V, Rossi D, Tabbo F, Ponzoni M, Chadburn A, Murty W, Bhagat G, Gaidano G, Inghirami G, Malek SN, Rabadan R, Dalla-Favera R: Genetics of follicular lymphoma transformation. Cell Rep 2014, 6:130-140.

41. Morin RD, Johnson NA, Severson TM, Mungall AJ, An J, Goya R, Paul JE, Boyle M, Woolcock BW, Kuchenbauer F, Yap D, Humphries RK, Griffith OL, Shah S, Zhu H, Kimbara M, Shashkin P, Charlot JF, Tcherpakov M, Corbett R, Tam A, Varhol R, Smailus D, Moksa M, Zhao Y, Delaney A, Qian H, Birol I, Schein J, Moore R, et al: Somatic mutations altering EZH2 (Tyr641) in follicular and diffuse large B-cell lymphomas of germinal-center origin. Nat Genet 2010, 42:181-185.

42. Green MR, Gentles AJ, Nair RV, Irish JM, Kihira S, Liu CL, Kela I, Hopmans ES, Myklebust JH, Ji H, Plevritis SK, Levy R, Alizadeh AA: Hierarchy in somatic mutations arising during genomic evolution and progression of follicular lymphoma. Blood 2013, 121:1604-1611.

43. Mar BG, Bullinger LB, McLean KM, Grauman PV, Harris MH, Stevenson K, Neuberg DS, Sinha AU, Sallan SE, Silverman LB, Kung AL: Lo Nigro L, Ebert $\mathrm{BL}$, Armstrong SA: Mutations in epigenetic regulators including SETD2 are gained during relapse in paediatric acute lymphoblastic leukaemia. Nat Commun 2014, 5:3469.

44. Mullighan CG, Zhang J, Kasper LH, Lerach S, Payne-Turner D, Phillips LA, Heatley SL, Holmfeldt L, Collins-Underwood JR, Ma J, Buetow KH, Pui CH, Baker SD, Brindle PK, Downing JR: CREBBP mutations in relapsed acute lymphoblastic leukaemia. Nature 2011, 471:235-239.

45. Oricchio E, Nanjangud G, Wolfe AL, Schatz JH, Mavrakis KJ, Jiang M, Liu X, Bruno J, Heguy A, Olshen AB, Socci ND, Teruya-Feldstein J, Weis-Garcia F, Tam W, Shaknovich R, Melnick A, Himanen JP, Chaganti RS, Wendel HG: The Eph-receptor A7 is a soluble tumor suppressor for follicular lymphoma. Cell 2011, 147:554-564.

46. Davis RE, Ngo VN, Lenz G, Tolar P, Young RM, Romesser PB, Kohlhammer H, Lamy L, Zhao H, Yang Y, Xu W, Shaffer AL, Wright G, Xiao W, Powell J, Jiang JK, Thomas CJ, Rosenwald A, Ott G, Muller-Hermelink HK, Gascoyne RD, Connors JM, Johnson NA, Rimsza LM, Campo E, Jaffe ES, Wilson WH, Delabie J, Smeland EB, Fisher Rl, et al: Chronic active B-cell-receptor signalling in diffuse large B-cell lymphoma. Nature 2010, 463:88-92.

47. Fowler N, Davis E: Targeting B-cell receptor signaling: changing the paradigm. Hematology Am Soc Hematol Educ Program 2013, 2013:553-560.

48. Li H, Durbin R: Fast and accurate short read alignment with Burrows-Wheeler transform. Bioinformatics 2009, 25:1754-1760.

49. NCBI database BioProject PRJNA240335. [http://www.ncbi.nlm.nih.gov/ bioproject/?term=PRJNA240335]

50. NBCl database BioProject PRJNA240336. [http://www.ncbi.nlm.nih.gov/ bioproject/?term=PRJNA240336]

\section{doi:10.1186/s13059-014-0432-0}

Cite this article as: Jiang et al:: Deep sequencing reveals clonal evolution patterns and mutation events associated with relapse in B-cell lymphomas. Genome Biology 2014 15:432.

\section{Submit your next manuscript to BioMed Central and take full advantage of:}

- Convenient online submission

- Thorough peer review

- No space constraints or color figure charges

- Immediate publication on acceptance

- Inclusion in PubMed, CAS, Scopus and Google Scholar

- Research which is freely available for redistribution

Submit your manuscript at www.biomedcentral.com/submit
C) BioMed Central 